\title{
Integration of a flow-type chemiluminescence detector on a glass electrophoresis chip
}

\author{
Rongguo Su ${ }^{a}$, Jin-Ming Lin ${ }^{\mathrm{a}, *}$, Katsumi Uchiyama ${ }^{\mathrm{b}}$, Masaaki Yamada ${ }^{\mathrm{b}}$ \\ ${ }^{a}$ Key Laboratory of Environmental Chemistry and Ecotoxicology, Research Center for Eco-Environmental Sciences, \\ Chinese Academy of Sciences, P.O. Box 2871, Beijing 100085, China \\ ${ }^{\mathrm{b}}$ Department of Applied Chemistry, Tokyo Metropolitan University, 1-1 Minami-Ohsawa, Hachioji, Tokyo 192-0397, Japan
}

Received 22 March 2004; received in revised form 28 April 2004; accepted 30 April 2004

Available online 15 June 2004

\begin{abstract}
A glass electrophoresis microchip integrated a flow-type chemiluminescence (CL) detection cell has been developed and evaluated. The chip pattern is a double-T-type electrophoretic sample injection and separation combining with a Y-type chemiluminecent detector. The double-T geometry allows for high-efficiency sample injection and geometric definition of sample plug size. The branch of Y was used as CL reagent channel, and the $\mathrm{CL}$ reagent was delivered by a lab-made micropump. Bis[(2,4,6-trichlorophenyl)]oxalate- $\mathrm{H}_{2} \mathrm{O}_{2} \mathrm{CL}_{\text {system was employed }}$ to detect dansyl amino acids. On this microchip, dansyl-phenylalanine and -sarcosine were successfully separated by electrophoresis and detected within $250 \mathrm{~s}$. The detection limits $(\mathrm{S} / \mathrm{N}=3)$ of dansyl-phenylalanine and -sarcosine could reach to 2.8 and $3.2 \mu \mathrm{M}$, respectively, due to the vigorous dilution of sample with CL reagent and timely removal of the waste solution from reaction area.
\end{abstract}

(C) 2004 Elsevier B.V. All rights reserved.

Keywords: Microchip electrophoresis; Chemiluminescence detection; Flow-type detection cell; Double-T injection

\section{Introduction}

Miniaturized total analysis system ( $\mu$-TAS), known also as "lab-on-a-chip" devices, can dramatically innovate the way chemical and biochemical assays are performed [1,2]. Over the past decade, $\mu$-TAS has experienced the phenomenal success and displayed enormous practical potentials $[3,4]$. These devices can integrate diversely functional units to accomplish screening of large sample populations or processing of special kinds of samples. It offers a new way to achieve fast analysis with high efficiency and low cost in a miniaturized setup including sample treatment, sample detection, collection processes and so on. The most noteworthy is possibly that this microtechnology has been applied to genomics [5,6], proteomics [7] and metabolomics [8].

The functional units of $\mu$-TAS including precisely defined wells, channels, and other features, which form the basis of the chip's sample holding, transfer, and separation operations, are usually fabricated on the $10-150 \mu \mathrm{m}$

\footnotetext{
* Corresponding author. Tel.: +8610 62841953; fax: +86 1062841953 .

E-mail address: jmlin@mail.rcees.ac.cn (J.-M. Lin).
}

scale and consistent with miniaturization and integration $[1,2]$. However, to realize miniaturization and integration of lab-on-a-chip devices, it is indispensible to employ equally miniaturized detectors. Up to now, laser-induced fluorescence (LIF) $[9,10]$ and mass spectroscopy (MS) [11,12] have been most often utilized for $\mu$-TAS detection because of their high sensitivity. Although submicromolar detectability can be readily obtained with these two types of detectors, the high cost and large size of the instruments are quite incompatible with the concept of $\mu$-TAS. Conventional photolithographic techniques can be conveniently used to fabricate micrometer-sized electrochemical (EC) electrodes directly on the substrate plates of diverse materials. Conducting metal films, as EC electrodes, were formed by sputtering, vapor deposition or chemical plating. Many types of EC electrodes have been successfully integrated on glass or polymer chips [13-15]. However, EC detection is prone to suffer from the high separation electric field, and the interference gives rise to a larger background current and a shifted redox potential.

Chemiluminescence (CL) has been shown to be a highly sensitive method for the detection in conventional capillary electrophoresis (CE) [16,17]. CL can be defined as the 
emission of light (ultraviolet, visible or infrared) from a molecule or atom in an electronically excited state produced by a chemical reaction [17]. It needs no light sources, and thus significantly reduces noise and eliminates Rayleigh and Raman scattering, allowing photon detectors to be operated at high gains to improve the ratio of signal to noise. Besides, there are more advantages in CL detection, such as wide dynamic range, simple instrumentation and versatility of use. Many CL systems have been well characterized and coupled to CE including peroxyoxalate [18], luminol [19], and barium peroxide [20]. Since these characters of CL match the demands of miniaturization and integration, CL will be a promising detection method for $\mu$-TAS. Mangru and Harrison [21] first realized CL detection on a microchip, Y-shaped reaction zone junction was adopted. Horseradish peroxidase (HRP) and fluorescein-conjugated HRP were separated and detected with a luminol CL system. Recently, Liu et al. [22] took the similar layout and scheme to successfully determinate the metal ions with luminol CL system on a poly(dimethylsiloxane) (PDMS) microchip. In these two reports, the separation channel had almost the same size with the CL reagent channel, so no excessive CL reagent was delivered, which was unfavourable to detection sensitivity. In addition, the $C L$ reagent was delivered by electroosmotic flow, as a result, some CL systems could not be employed when CL reagent was only dissolved in organic solvent. The end-channel batch-type detection cell for CL detection on microchip was also demonstrated by Hashimoto et al. [23] Dansyl amino acids were monitored using a peroxyoxalate CL system. Since the detection cell also worked as the waste reservior, the waste produced by the $\mathrm{CL}$ reaction was accumulated in the detection cell, which would have a negative influence on the sensitivity and reproducibility.

Consequently, our efforts have been directed toward the development of lab-on-a-chip with on-line CL detection that are consistent with the wide range of possible applications for these devices. In this paper, a microchip integrated with $\mathrm{CL}$ detector was described. A flow-type detection cell was adopted toward two objectives: high sensitivity and wide range of applications.

\section{Experimental}

\subsection{Chemicals}

Tris(hydroxymethyl)aminomethane (Tris) and boric acid were purchased from Beijing Chemical Reagents Co., Ltd. (Beijing, China). The separation buffer was $0.1 \mathrm{M}(\mathrm{pH}$ 7.0) tris-borate. A $1.0 \mathrm{mM}$ stock solution of bis(2,4,6trichlorophenyl)oxalate (TCPO) (Acros, USA) was prepared in acetonitrile (Tianjin Shield Company, China). CL reagent was obtained by mixing $5.0 \mathrm{~mL}$ of $1.0 \mathrm{mM}$ TCPO stock solution and $29.0 \mu \mathrm{L} 30 \% \mathrm{H}_{2} \mathrm{O}_{2}$ (Shantou Xilong Chemical Co., Ltd. China) prior to use. Dansyl-phenylalanine and -sarcosine (Sigma, St. Louis, MO) were dissolved in

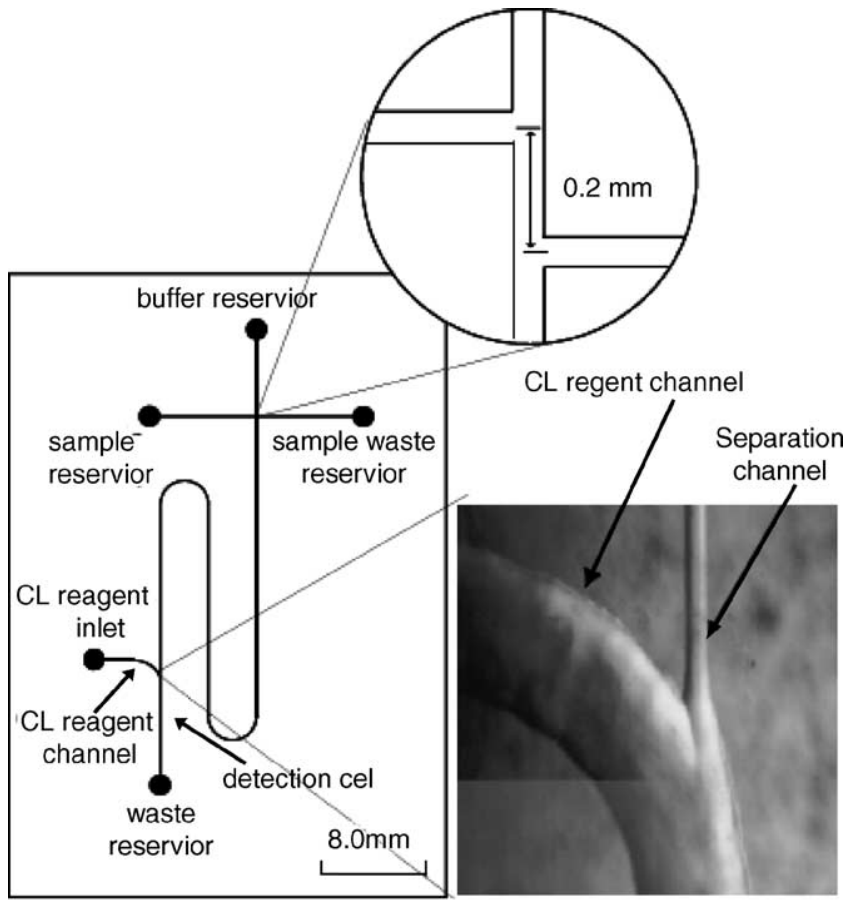

Fig. 1. Schematic diagram of the microchip.

running buffer. Water, purified by a Barnstead EASYpure LF system (Dubuque, IA, USA), was used for all solutions. All chemicals were of analytical grade and used without further purification.

\subsection{Microchip device}

The microchip used here, illustrated in Fig. 1, was fabricated from soda lime glass (Shaoguang Microelectronics Co., China) using standard photolithography, wet chemical etching, and heat bonding techniques [24]. The Y-shaped layout conbining with double-T injection mode was adopted. The channel length is $10.0 \mathrm{~mm}$ from the samlpe reservoir to the injection cross, $10.0 \mathrm{~mm}$ from the sample waste reservoir to the injection cross, $10.0 \mathrm{~mm}$ from the buffer reservoir to the injection cross, and $90.0 \mathrm{~mm}$ from the injection cross to the detection cell. The detection cell is $10.0 \mathrm{~mm}$ long. The radius of the three turns including the turn of the CL reagent channel is $2.5 \mathrm{~mm}$. The dimensions of the channels are $40 \mu \mathrm{m}$ deep and $80 \mu \mathrm{m}$ wide except that the CL reagent channel and detection cell are $600 \mu \mathrm{m}$ deep and $800 \mu \mathrm{m}$ wide. Other details were depicted in Fig. 1. The glass substrates were purchased precoated with a thin chrome layer and positive AZ1350 photoresist. The top glass substrate contained the channels and reservoir openings. Patterns of channels and reserviors were first designed with Adobe Illustrator (Adobe Systems Inc. USA) and transferred onto a transparent film with a laser phototypesetting system (3556 dpi, MAKO Imagesetter, MAKO 4650, ECRM, USA). These patterns were then transferred onto the photoresist layer by a UV light exposure. After the exposed photoresist 
and corresponding chromium layer were removed, a wet chemical etching was carried out with a etchant $\left(\mathrm{HF}: \mathrm{NH}_{4} \mathrm{~F}\right.$, $0.4 \mathrm{M}: 0.4 \mathrm{M})$. The etching process included two steps: (a) etching channels and reservoirs, the back of the glass plate was protected with adhensive tape, then it was immersed in the etchant; (b) etching detection cell and CL reagent channel, when the separation and injection channels were etched to the depth designed, they were also protected with adhensive tape, then the detection cell and CL reagent channel were etched again with $0.8 \mathrm{M}$ etchant till to the depth required. After the above treatment, the left photoresist and chromium layer were removed. The five reservoirs were then drilled using a minidriller (Sakai, Korea). The top and bottom substrates were cleaned and bonded as reported [24].

\subsection{Microchip operation}

High voltage was applied to the reservoirs of the microchip by a power supply (CDY-800L, Shanhua, Shandong Chemical Institue, China) with four independently controlled high-voltage outputs and two high-voltage relays. The two high-voltage relays were connected in series between the high-voltage supplies and the buffer and waste reservoirs to remove the high voltage at these reservoirs. Platinum wires $(\Phi=0.3 \mathrm{~mm})$ as electrodes were inserted into the reserviors. Before experiments, the channels were conditioned with $0.1 \mathrm{M}$ sodium hydroxide for $20 \mathrm{~min}$ to activate the surface, then rinsed with ultrapure water. To perform the separation, the channels and reservoirs were first filled with the buffer solution using a disposable syringe except for the waste reservior, then the buffer solution in the sample reservior was replaced by sample solution, and the buffer solution in the CL reagent channel and detection cell was replaced by $\mathrm{CL}$ reagent solution. It should be mentioned that the waste reservior was filled with CL reagent solution at half. In injection mode, a potential of $+1.0 \mathrm{kV}$ was applied to the sample reservoir for $15 \mathrm{~s}$ with the sample waste reservoir grounded and other reservoirs floating. In the run mode, the voltages applied to the buffer, sample waste, and waste reservoirs were $2.9,3.6,2.9$, and $0.0 \mathrm{kV}$, respectively. This corresponded to electric field strength of about $340 \mathrm{~V} \mathrm{~cm}^{-1}$ for separation channel. At the same time, the CL reagent solution was delivered at the rate of $15.0 \mu \mathrm{L} \mathrm{min}^{-1}$ through $\mathrm{CL}$ reagent channel by a lab-made micropump. The analyte was transported into the separation channel toward the detection cell, and then mixed with CL reagent solution at the junction of the detecton cell and separation channel. The potentials applied to the sample and sample waste reservoirs were used to prevent the sample solution in the injection channel from leaking into the separation channel. The back side of the microchip was packed with black adhesive tape on which a rectangular aperture $(1.2 \mathrm{~mm} \times 5.0 \mathrm{~mm})$ was made as the detection window and located just on the mixing point. The CL signal was recorded by the Ultra-Weak Luminescence Analyzer (Institute of Biophysics Academia Sina, China) located under the detection window.

\section{Results and discussion}

\subsection{Design of the microchip}

Fig. 1 provides the detailed images showing precisely the double-T junction and the location and orientation of the separation channel, CL ragent channel and detection cell. The injection system on a microfluidic chip is one of the key elements in the sample handling process, and its characteristics have a great influence on the performance of a microchip [25]. In the injection technology of microchip, the cross mode has been commonly used [26,27]. Generally, this injection system is only capable of delivering a fixed-volume sample plug into the separation channel. The volume of the sample plug is determined by the geometry of the cross-sectional area of the injection channel. Therefore, this configuration is clearly unsuitable for an application that requires the injection of larger sample plugs to enhance detection sensitivity, which is largely dependent on the volume of sample solution. A more appropriate solution is to replace the cross-channel arrangement with double- $\mathrm{T}$ form [28]. This geometry allows for high-efficiency sample injections and geometric definition of sample plug size, irrespective of injector loading times or the applied injection potentials $[28,29]$. The injected volume with the double-T-form injection system is determined by the double-T's length and always larger than that with the cross-injection system [25]. Generally in CE, separation efficiency and resolving power are improved by increasing the field strength. However, most separations have a field strength limit, above which Joule heating will begin to noticeably degrade the separation efficiency. In addition, the separation efficiency and the resolving power may be further enhanced by lengthening the separation channel while keeping the field strength constant. This will result in a linear increase in plates and a square root increase in resolution with increasing channel length. Often this design requires several turns to be introduced into separation channel [30]. Unfortunately, the introduction of turns into the separation channel will result in an elongation of the band, and thus decrease the separation efficiency. However, this negative influence can be partly reduced by increasing the turn radius of curvature and adopting complementary pairs of turns [31]. For this reason, a complementary pair of $180^{\circ}$ turns with the radius of $2.5 \mathrm{~mm}$ was employed on the microchip used here. The turn on the CL reagent channel was one quarter of a round with radius of $2.5 \mathrm{~mm}$, so the $\mathrm{CL}$ reagent flowed in the same direction with the electroosmotic flow from the separation channel and the effect of pressure-driven flow on electroosmotic flow was decreased. The CL reagent was delivered by a micropump. Because of the strong dependence on local channel structures and material as well as fluid composition, electroosmotic flow is frequently accompanied by instability. However, the pressure-driven flow can provide a stable and controllable flow velocity, more importantly, some CL systems, although no sufficient EOF is produced to deliver CL reagents, can 
be employed on the microchip. The CL reagent channel and detection cell have about ten times dimensions of separation channel, which made the CL reagent superfluous, and thus the detection sensitivity is enhanced. Furthermore, the larger CL reagent channel and detection cell are compatible with the micropump and have a significantly lower resistance to flow than the separation channel and also reduce the effects of pressure-driven flow on electroosmotic flow.

\subsection{Analytical performance}

This work aimed at developing an effective on-line CL detector for microchip. According to the reports of CL detection in CE [16], the achieved sensitivity is largely dependent on the two crucial keys: The first is the dynamics of the CL reaction. Only those with rapidly responding reaction dynamics could be successfully employed. The second is the configuration of the CL detector, that is, the way for mixing CL reagent and the analytes. The peroxyoxalate CL system has been widely applicable to detection of fluorescence and fluorescence-labeled compounds in CE $[18,32,33]$. TCPO was representative as a peroxyoxalate CL reagent here. The dansyl amino acids were determinated to evaluate the configuration of the microchip in terms of sensitivity, reproducibility, and resolution. Another important reason to choose the above-mentioned CL system was that TCPO only could be prepared in organic solvent that would be difficult to be delivered by electroosmotic flow.

\subsection{Mixing mode of the analytes and the chemiluminescent reagent}

Since CL detection requires CL reagent, the mixing mode of the analyte and the CL reagent has a marked influence on the light intensity. Different mixing modes might give rise to light quench, even under the same conditions. In conventional flow injection CL analysis (FIA) [34] and liquid chromatography CL analysis [35,36], TCPO stream and hydrogen peroxide stream mix in advance and then react with analytes. However, when sample injection is very small (i.e., nanoliters), such a mixing mode might lead to a significant decrease in sensitivity [19]. To be conveniently operated and improve the sensitivity, the mixed solution of TCPO and $\mathrm{H}_{2} \mathrm{O}_{2}$, used as the CL reagent, was introduced. In a mixture with hydrogen peroxide, TCPO was most stable when dissolved in acetonitrile. The TCPO was hydrolysed rapidly with water, which would result in a decrease in CL intensity [37]. Hydrogen peroxide is commercially available as a $30 \%$ water solution, and hence an increase in hydrogen peroxide concentration is accompanied by an increase in water concentration. So the concentration of hydrogen peroxide was set at $<150 \mathrm{mM}$, which gave enough CL intensity with a large signal to noise ratio, and the mixture was prepared just before use to prevent decomposition of TCPO. This mode has been successfully realized on capillary electrophoresis $[33,38]$. Consequently, the acetonitrile solution of $5.0 \mathrm{~mL} 1.0 \mathrm{mM}$ TCPO and $29.0 \mu \mathrm{L} 30 \% \mathrm{H}_{2} \mathrm{O}_{2}$ was chosen as the $\mathrm{CL}$ reagent. The $\mathrm{CL}$ reagent flow rate is also one of important factors that determine the performance of the lab-on-a-chip device. Based on the optimized
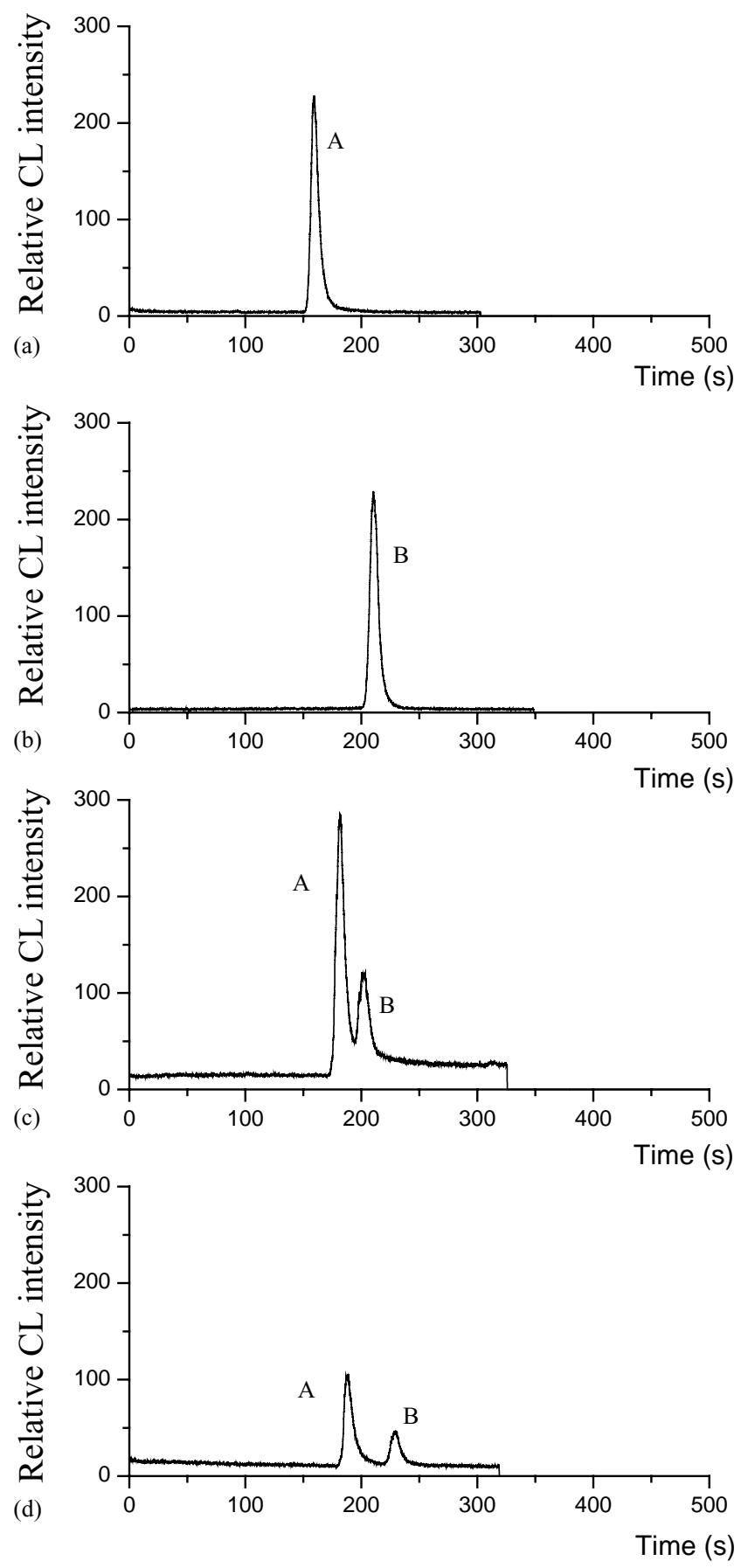

Fig. 2. Electropherograms of dansyl amino acids achieved with on-chip $\mathrm{CL}$ detection. Running buffer $0.1 \mathrm{M}$ Tris-borate $(\mathrm{pH} 7.0)$; $\mathrm{CL}$ reagent $\left(5.0 \mathrm{~mL} 1.0 \mathrm{mM}\right.$ TCPO solution $\left.+29.0 \mu \mathrm{L} 30 \% \mathrm{H}_{2} \mathrm{O}_{2}\right)$ at $15.0 \mu \mathrm{L}$ $\min ^{-1}$; sample injection at $1000 \mathrm{~V}$ for $15 \mathrm{~s}$; separation at $3600 \mathrm{~V}$ to buffer reservior, $2900 \mathrm{~V}$ to sample and sample waste reserviors, $0 \mathrm{~V}$ to waste reservior. (A) Dansyl-phenylalanine; (B) Dansyl-sarcosine. (a) $20 \mu \mathrm{M}$ dansyl-phenylalanine; (b) $20 \mu \mathrm{M}$ dansyl-sarcosine; (c) $20 \mu \mathrm{M}$ dansyl-phenylalanine and $10.0 \mu \mathrm{M}$ dansyl-sarcosine; (d) $4 \mu \mathrm{M}$ dansyl-phenylalanine and $2.0 \mu \mathrm{M}$ dansyl-sarcosine. 
Table 1

Migration times, peak height, theoretical plate numbers, and resolutions for repetitive runs

\begin{tabular}{|c|c|c|c|c|c|c|c|c|c|}
\hline \multirow[t]{2}{*}{ Number } & \multicolumn{4}{|l|}{ Peak 1} & \multicolumn{4}{|l|}{ Peak 2} & \multirow[t]{2}{*}{$R_{\mathrm{s}}$} \\
\hline & Time (s) & $\begin{array}{l}\text { Peak height } \\
\text { (relative CL intensity) }\end{array}$ & $W_{1 / 2}(\mathrm{~s})$ & $\begin{array}{l}\text { Theoretical plate } \\
\text { numbers }\end{array}$ & Time (s) & $\begin{array}{l}\text { Peak height } \\
\text { (relative CL intensity) }\end{array}$ & $W_{1 / 2}(\mathrm{~s})$ & $\begin{array}{l}\text { Theoretical } \\
\text { plate numbers }\end{array}$ & \\
\hline 1 & 180 & 290 & 8 & 2800 & 210 & 130 & 9 & 3010 & 1.3 \\
\hline 2 & 200 & 300 & 9 & 2730 & 230 & 120 & 12 & 2030 & 1.4 \\
\hline 3 & 190 & 340 & 12 & 1380 & 220 & 140 & 11 & 2210 & 1.2 \\
\hline R.S.D. (\%) & 6.1 & 8.5 & & & 4.5 & 7.7 & & & \\
\hline
\end{tabular}

Table 2

Linear ranges, detection limits of dansyl-phenylalanine and -sarcosine

\begin{tabular}{llll}
\hline Analyte & Linear range $(\mu \mathrm{M})$ & $r$ & Linear regression equation \\
\hline Dansyl-phenylalanine & $1.0-100.0$ & 0.9982 & $H=1.07 \times 10^{7} \mathrm{C}$ \\
Dansyl-sarcosine & $1.0-100.0$ & 0.9973 & $H=8.24 \times 10^{6} \mathrm{C}$ \\
\hline
\end{tabular}

$r$ : linear correlation coefficient; $H$ : peak height (relative CL intensity); $C$ : concentration of analytes (M).

experiments, the CL reagent flow rate of $15.0 \mu \mathrm{L} \mathrm{min}^{-1}$ was taken.

\subsection{Electrophoretic separation of dansyl-phenylalanine and-sarcosine}

Since Tris buffer can catalyze the TCPO- $\mathrm{H}_{2} \mathrm{O}_{2} \mathrm{CL}$ reaction [36] and Tris buffer is also suitable for separating dansyl amino acids on microchip capillary electrophoresis (MCE) [23], the 0.1 M Tris-borate ( $\mathrm{pH} 7.0)$ solution was used as electrophoresis buffer.

The volume of the analyte plug moving in the separation channel was by far small enough compared to the volume of the reagent flowing in the CL reagent channel; therefore, the optimum solvent environment for CL reaction can be obtained by maintaining nonaqueous conditions on the point of detection, for this CL system was mainly dependent on the nonaqueous environment [33]. Under above conditions, dansyl-phenylalanine and -sarcosine were separated and detected on this microchip. Electropherograms were obtained, as shown in Fig. 2. Fig. $2 \mathrm{a}$ and $\mathrm{b}$ are the electrophoregrams of $20.0 \mu \mathrm{M}$ dansyl-phenylalanine and $20.0 \mu \mathrm{M}$ dansyl-sarcosine, respectively. Fig. 2c (20.0 $\mu \mathrm{M}$ dansyl-phenylalanine and $10.0 \mu \mathrm{M}$ dansyl-sarcosine) and Fig. $2 \mathrm{~d}$ (4.0 $\mu \mathrm{M}$ dansyl-phenylalanine and $2.0 \mu \mathrm{M}$ dansylsarcosine) are the separation electropherograms achieved on this lab-on-a-chip system. It is indicated that dansylphenylalanine and -sarcosine were successfully separated and detected within $250 \mathrm{~s}$. A peak width at half-height of about $10 \mathrm{~s}$ was obtained. Unlike batch-type CL detectors, after the analyte coming into contact with "fresh" reagents, the mixture was quickly removed from detectable reaction area, thus reduced the peak tailing and sharpen the peak, because the signal from the residual CL reaction out of detection window was not recorded. Some reports on CE-CL [32] and MCE [23] have mentioned the main shortcoming of the batch-type CL detection cell, a relatively low number of theoretical plates due to the band broadening resulting from the postcapillary mixing of analytes and the corresponding CL reagents. In this experiment, the average theoretical plate number reached 3000 .

Under the same conditions, we performed repetitive runs of mixture samples of $20.0 \mu \mathrm{M}$ dansyl-phenylalanine and $10.0 \mu \mathrm{M}$ dansyl-sarcosine without exchange of the solutions in each reservoir between runs. Table 1 represents results for migration time, peak height, theoretical plates, and resolution concerning three repetitive runs. Theoretical plates decreased with the broadening of peaks.

\subsection{The linear range and detection limits}

Linear relation could be achieved between concentration and peak height for both the compounds. The linear ranges and detection limits of dansyl-phenylalanine and -sarcosine are shown in Table 2.

According to experiments, the detection limits of the analytes based on a peak height of three times the baseline noise are in the linear ranges. After the $H$ of the linear regression equations were replaced by three times the baseline noise (got by running the electrophoresis chip system without injection of analytes), the detection limits of dansylphenylalanine and-sarcosine were obtained. Compared with on-chip batch-type CL detectors [23], the detection sensitivity of this apparatus has been improved, maybe due to the vigorous dilution of sample with CL reagent and timely removal of the waste from reaction area.

\section{Conclusions}

CL detection system has many advantages: high sensitivity and selectivity; a simple, cheap optical system requiring no light source, which match the demands of $\mu$-TAS. The microchip integrated with CL detection was realized here. The double-T injection mode allowed the large volume of 
sample solution injected, and thus the concentration detection limit was significantly decreased. Since the detection cell was flow-type and the CL reagent was delivered with a micropump, this microchip can be used for almost all of CL systems. The success of this microchip system showed that CL is a most promising detection method for $\mu$-TAS in chemical and biochemical analysis.

\section{Acknowledgements}

This work was supported by National Natural Science Foundation of China (No. 50273046) and National Science Fund for Distinguished Young Scholars of China (No. 20125514).

\section{References}

[1] D.R. Reyes, D. Iossifidis, P.-A. Auroux, A. Manz, Anal. Chem. 74 (2002) 2623.

[2] P.-A. Auroux, D. Iossifidis, D.R. Reyes, A. Manz, Anal. Chem. 74 (2002) 2637

[3] A.B. Jemere, R.D. Oleschuk, D.J. Harrison, Electrophoresis 24 (2003) 3018.

[4] A.C. Henry, T.J. Tutt, M. Galloway, Y.Y. Davidson, C.S. McWhorter, S.A. Soper, R.L. McCarley, Anal. Chem. 72 (2000) 5331.

[5] A.T. Woolley, G.F. Sensabaugh, R.A. Mathies, Anal. Chem. 69 (1997) 2181.

[6] M.A. Northrup, B. Benett, D. Hadley, P. Laudre, S. Lehew, J. Richards, P. Stratton, Anal. Chem. 70 (1998) 918.

[7] D. Figeys, R. Aebersold, Anal. Chem. 70 (1998) 3721.

[8] J. Wang, M.P. Chatrathi, B. Tian, R. Polsky, Anal. Chem. 72 (2000) 2514.

[9] G. Ocvirk, T. Tang, D.J. Harrison, Analyst 123 (1998) 1429.

[10] W. Xu, K. Uchiyama, T. Shimosaka, T. Hobo, J. Chromatogr. A 907 (2001) 279.

[11] L. Licklider, X.-Q. Wang, A. Desai, Y.-C. Tai, T.D. Lee, Anal. Chem. 72 (2000) 367.

[12] B. Zhang, F. Foret, B.L. Karger, Anal. Chem. 73 (2001) 2675.
[13] R.S. Martin, A.J. Gawron, S.M. Lunte, C.S. Henry, Anal. Chem. 72 (2000) 3196.

[14] B. Graß, A. Neyer, M. Jühnck, D. Siepe, F. Eisenbeiß, G. Weber, R. Hergenröder, Sens. Actuators B 72 (2001) 249.

[15] R.P. Baldwin, T.J. Roussel Jr., M.M. Crain, V. Bathlagunda, D.J. Jackson, J. Gullapalli, J.A. Conklin, R. Pai, J.F. Naber, K.M. Walsh, R.S. Keynton, Anal. Chem. 74 (2002) 3690.

[16] X.-J. Huang, Z.-L. Fang, Anal. Chim. Acta 414 (2000) 1.

[17] L.J. Kricka, Anal. Chem. 71 (1999) 305R.

[18] N. Wu, C.W. Huie, J. Chromatogr. 634 (1993) 309.

[19] B. Huang, J.-J. Li, L. Zhang, J.-K. Cheng, Anal. Chem. 68 (1996) 2366.

[20] J.-M. Lin, H. Goto, M. Yamada, J. Chromatogr. A 844 (1999) 341.

[21] S.D. Mangru, D.J. Harrison, Electrophoresis 19 (1998) 2301.

[22] B.-F. Liu, M. Ozaki, Y. Utsumi, T. Hattori, S. Terabe, Anal. Chem. 75 (2003) 36.

[23] M. Hashimoto, K. Tsukagoshi, R. Nakajima, K. Kondo, A. Arai, J. Chromatogr. A 867 (2000) 271.

[24] Z.H. Fan, D.J. Harrison, Anal. Chem. 66 (1994) 177.

[25] L.-M. Fu, R.-J. Yang, G.-B. Lee, H.-H. Liu, Anal. Chem. 74 (2002) 5084.

[26] S.C. Jacobson, R. Hergenröder, L.B. Koutny, R.J. Warmack, J.M. Ramsey, Anal. Chem. 66 (1994) 1107.

[27] S.V. Ermakov, S.C. Jacobson, J.M. Ramsey, Anal. Chem. 72 (2000) 3512.

[28] K. Fluri, G. Fitzpatrick, N. Chiem, D.J. Harrison, Anal. Chem. 68 (1996) 4285.

[29] L.L. Shultz-Lockyear, C.L. Colyer, Z.H. Fan, K.I. Roy, D.J. Harrison, Electrophoresis 20 (1999) 529.

[30] S.-S. Park, S. Cho II, M.-S. Kim, Y.-K. Kim, B.-G. Kim, Electrophoresis 24 (2003) 200.

[31] C.T. Culbertson, S.C. Jacobson, J.M. Ramsey, Anal. Chem. 70 (1998) 3781 .

[32] K. Tsukagoshi, Y. Obata, R. Nakajima, J. Chromatogr. A 971 (2002) 255.

[33] K. Tsukagoshi, Y. Okumura, R. Nakajima, J. Chromatogr. A 813 (1998) 402.

[34] K. Honda, J. Sekino, K. Imal, Anal. Chem. 55 (1983) 940.

[35] K.W. Sigvardson, J.M. Kennish, J.W. Birks, Anal. Chem. 56 (1984) 1096.

[36] K.W. Sigvardson, J.W. Birks, J. Chromatogr. 316 (1984) 507.

[37] N. Imaizumi, K. Hayakawa, M. Miyazaki, Analyst 114 (1989) 161.

[38] K. Tsukagoshi, T. Kimura, T. Fuji, R. Nakajima, A. Arai, Anal. Sci. 17 (2001) 345. 